\section{SMOKING AND PERSONALITY*}

BY

\author{
H. J. EYSENCK, Ph.D.
}

Institute of Psychiatry, London

MOLLIE TARRANT, B.A. MYRA WOOLF, B.Sc.(Econ.)

AND

\section{ENGLAND}

Mass Observation

The discovery of a statistical association or correlation between lung cancer and smoking (Doll and Hill, 1952, 1954 ; Hammond and Horne, 1954 ; Stocks and Campbell, 1955 ; Berkson, 1958) has led some investigators to put forward the hypothesis that a causal relationship exists between these two factors. This hypothesis has been severely criticized by Fisher (1959), Berkson (1958), and others, on the grounds that alternative and equally plausible theories existed and had not been disproved. These critics further pointed to various weak points in the causal argument, such as the fact that inhaling does not aggravate the alleged effects of smoking, but, on the contrary, appears to have, if anything, an ameliorating effect ; that pipe smoking does not appear to have the same effects as cigarette smoking; or that smoking has a statistical association not only with cancer but also with other diseases which had never been conceived to have the same aetiology as cancer.

The main hypothesis which has been put forward to account for the correlation, other than that of direct causation, has been Fisher's suggestion "that a common cause supplies the explanation"-that is, that both rate of smoking and proneness to lung cancer are due to some third variable underlying both; "the obvious common cause to think of is the genotype." He goes on to say that "if there is any genotypic difference between the different smoking classes we may expect differences in the type or frequency of the cancers that they display." Why, he asks, do some people take to the pipe rather than to cigarettes, while others make the opposite choice? "Because they are made that way." In other words, innate personality features determine our behaviour with respect to smoking, at least in part, and they also determine our proneness to lung cancer. In spite of the fact that there is no real evidence in favour of this hypothesis, it cannot be dismissed out of hand; it suggests new experiments to throw more light on possible genotypic differences between different groups of smokers. It was with the intention of supporting or confirming certain hypotheses related to this general theory that the present research was undertaken.

\section{Theoretical Analysis}

A survey of the literature disclosed little that was of help in planning the investigation (McArthur, Waldron, and Dickinson, 1958), and accordingly recourse was had to theoretical considerations related to the general

*In the summer of 1958 Mass-Observation Ltd. was commissioned by the Tobacco Manufacturers' Standing Committee to carry out a survey to test certain hypotheses on the personality characteristics of smokers and non-smokers suggested by Professor H. J. Eysenck. The main results of the study (Mass-Observation. 1959) are summarized in this paper. dimensional theory of personality (Eysenck, 1947, 1952, 1957, 1960a, 1960b). Three main hypotheses were advanced, as well as two minor ones. The terms used in stating these hypotheses (" extraversion," "neuroticism," "rigidity") have been defined in terms of this theory and are not here redefined; the reader is referred to the references just cited for a detailed account.

According to the first hypothesis, smoking habits should be related to extraversion in the sense that the number of cigarettes smoked would increase with degree of extraversion. This hypothesis was derived from the well-known characteristic of the extraverted personality to concentrate on objects in the outer world, in contrast to the introvert, who tends to be preoccupied with his own thought processes and other internal states. According to this hypothesis one would expect extraverts to be particularly heavy consumers not only of cigarettes but also of alcohol, sweets, and other pleasure-giving objects in the external world.

The second hypothesis was to the effect that the more emotionally unstable, neurotic type of person would be the heavier smoker because smoking, like many other motor and sensory habits, reduces the strength of an aroused emotion. Consequently, for a person of this type cigarettes would become a solace and might almost be regarded in the light of a medicine.

The third general hypothesis investigated related to the personality trait of rigidity, where it was postulated that the more rigid person would be less likely to smoke. This was deduced from the fact that smoking, as indeed all pleasurable activities, tends to be regarded as slightly sinful, and would therefore tend to be shunned by the rigid, puritanically minded type of person.

\section{The Experiment}

\section{The Questionary}

To test these hypotheses, a questionary was constructed which contained questions which in previous work had been found to be good, reliable, and reasonably valid measures of these personality traits (Eysenck, 1959 ; Nigniewitzky, 1955); this questionary is printed in the Appendix (p. 1460).

In addition to these general hypotheses, two items were included to test quite specific hypotheses; to wit, items $7 \mathrm{~g}$ and $8 \mathrm{~g}$-that is, $7 \mathrm{~g}$ : Do you get so excited that you use your hands when you talk ? and $8 \mathrm{~g}$ : Do you have any habits like chewing pencils or biting fingernails or things like that ? As regards $7 \mathrm{~g}$, use of hands when talking, the hypothesis was that the natural impulse of people to use their hands would be somewhat inhibited by having a cigarette as an alternative outlet, so that non-smokers would use their hands more. Similarly, it was assumed that non-smokers would be more likely to chew pencils and bite their fingernails than smokers, who, after all, have an alternative outlet.

\section{Sample Tested}

The design of the experiment makes use of 24 groups of subjects divided equally on the basis of age (40-59 and 60-70), class (ABC and $\mathrm{DE}$ ), and smoking habits (non-smokers, light, medium, and heavy smokers, pipe smokers, and ex-smokers). The hypotheses only deal with the relative positions of four groups-namely, the non-smokers and the light, medium, and heavy smokers : no prediction could be made with respect to ex-smokers 
or pipe smokers. There are 100 subjects in each of the groups except among the older non-smokers; for purposes of analysis, scores in these groups have been pro-rated so as to be comparable in all respects with the other groups.

The object of the sample design was to obtain an analytic (as opposed to a representative) sample of men. For the purposes of analysis, it was decided to interview 400 men in each of six smoking groups (light, medium, and heavy cigarette smoker, pipe smoker, non-smoker, and ex-smoker) so that, within each smoking group, there were 100 men in each of two classes and two age-groups.

In detail the requirements were as shown in Table $I$.

TABLE I

\begin{tabular}{ll|c|c|c|c}
\hline & & \multicolumn{2}{|c|}{ Class ABC } & \multicolumn{2}{c}{ Class DE } \\
\cline { 3 - 5 } & & $40-59$ & $60-70$ & $40-59$ & $60-70$ \\
\hline Light &. & 100 & 100 & 100 & 100 \\
Medium &. & 100 & 100 & 100 & 100 \\
Heavy &. & 100 & 100 & 100 & 100 \\
Pipe.. &. & 100 & 100 & 100 & 100 \\
Ex-.. &. & 100 & 100 & 100 & 100 \\
Non- &. & 100 & 100 & 100 & 100 \\
\hline
\end{tabular}

For each subgroup the selection of informants was to be randomized as much as possible, though the sampling method used was quota sampling.

In the final event, it proved impossible within the time and cost limits of the inquiry to contact 100 non-smokers in the older age-groups, and the numbers analysed were 70 in Class ABC and 90 in Class DE. To obtain the numbers required for the experimental sample, the approximate number of contacts finally made was over 7,000 .

A three-stage sampling design was employed. At the first two stages the method of selection of units was random. The general plan was as shown in Table II.

TABLE II

\begin{tabular}{|c|c|c|}
\hline Stage & Stratification Factors & Notes \\
\hline $\begin{array}{l}\text { 1. Administrative } \\
\text { districts } \\
\text { 2. Wards }\end{array}$ & $\begin{array}{l}\text { (a) Geographical region (6) } \\
\text { (b) Conurbation/urban/rural } \\
\text { (c) J-index } \\
\text { J-index }\end{array}$ & $\begin{array}{l}\text { Selection of individual } \\
\text { areas within each stra- } \\
\text { tum was made with a } \\
\text { probability propor- } \\
\text { tionate to its size }\end{array}$ \\
\hline 3. Individuals & $\begin{array}{l}\text { Quota controls: } \\
\text { (a) Age } \\
\text { (b) Class } \\
\text { (c) Occupation }\end{array}$ & \\
\hline
\end{tabular}

Stage 1. -100 administrative districts were selected as first-stage units. Before selection, the districts were stratified into six geographical regions and three urban/ rural breakdowns. Within each stratum, the administrative districts were listed in order of their J-index, and the list was divided into two groups of approximately equal size. An equal number of administrative districts were selected from each part of the list, and the selection of individual administrative districts was made with a probability proportionate to its size. As no J-index exists for areas in Scotland, the selection of administrative districts within each geographical/urban, etc., stratum was made randomly.

Stage 2.-The second stage of sampling was to select three wards from each of the larger (population 50,000+) administrative districts selected at the first stage. In the smaller administrative districts a $100 \%$ sample-that is, all the wards within each administrative districtwere selected for investigation. Selection of individual wards was made in a way similar to the selection of administrative districts. The wards within each district were listed in order of their J-index, and the list was divided into three groups of approximately equal size. One ward was selected from each group. It is not always usual, when using the method of quota sampling, to use a second sampling stage. Generally, interviewers are instructed to spread their interviews over the whole of the first-stage unit, whatever it might be. For this survey, however, it was thought preferable to provide some method of making certain that the interviews were not clustered in one part of the larger administrative districts, and this was done by selecting second-stage units which ensured that the interviews were carried out in three different parts of the towns concerned.

Stage 3.-At the final stage the selection of individuals to be interviewed was not made randomly, but by the method of quota sampling. Interviewers were allocated quotas on age, social class, and smoking group, so that information was obtained from the required respondents. A looser control was placed on occupation so that the distributive trades were not overrepresented, as tends to be the case with quota sampling.

\section{Allocation of Subjects}

Subjects were allocated to one of six groups on the following basis.

Light smokers were taken to be those who said that they smoked 14 or fewer cigarettes daily.

Medium smokers were taken to be those who said that they smoked between 15 and 24 cigarettes daily.

Heavy smokers were taken to be those who said that they smoked 25 or more cigarettes daily.

Pipe smokers were taken to be those who smoked a pipe only, or, if they had more than one smoking outlet, accounted for more than three-quarters of their total consumption in pipe tobacco.

Ex-smokers were taken to be those who did not smoke at present but who claimed that, in the past, they had smoked at a rate equivalent to more than one cigarette a day or $2 \mathrm{oz}$. (57 g.) of tobacco in two months for at least as long as a year.

Non-smokers were those who did not smoke at present and claimed that in the past they had not smoked more than one cigarette a day or more than $2 \mathrm{oz}$. $(57 \mathrm{~g}$.) of tobacco for at least as long as a year.

Throughout, a conversion factor of $1 \mathrm{oz}$. $(28.3 \mathrm{~g}$.) of tobacco as the equivalent of 30 cigarettes was used. This factor was used to categorize those who hand-rolled their cigarettes, into light, medium, and heavy cigarette smokers, and also to assess the relative strength of pipe and cigarette smoking among those who smoked both. Those whose consumption of tobacco was at least threequarters in pipe tobacco "were classified as "pipe" smokers ; those whose consumption was at least threequarters in cigarettes were classified as cigarette smokers in the relevant category of light, medium, or heavy, depending on the number of cigarettes smoked. Those in between these two groups-that is, those who smoked about an equal amount in both pipes and cigaretteswere omitted from the sample.

Unreliability of self-assessments may serve to reduce the final relationships discovered, but it has been shown that the reliability of such statements about smoking habits as were called for by the present research design 
is reasonably high (Todd and Laws, 1958) ; it is doubtful if the fact that self-assessments were asked for constitutes a major weakness of this study.

\section{Results}

\section{Individual Questions}

The first analysis to be carried out was done on each question separately. Scores were derived by giving two points to each "Yes" answer and one point to each "?"; no points were given to "No" answers. An analysis of variance was then carried out for each of the questions, both for main effects and for interactions. In this way it was possible to discover for each question whether the answers showed significant differences associated with class, age, or smoking habits (main effects); and it was also possible to determine whether there was any interaction between some of these main effects, such for instance as is described later in connexion with question $7 \mathrm{~g}$, where it was found that the tendency to use one's hands while talking was indeed associated significantly with non-smoking, but only in the lower social groups. The analysis revealed that age was much the most important variable in determining response differences, and that class was relatively unimportant. Smoking habits uncomplicated by interaction effects appeared significant in connexion with five questions $(6 \mathrm{f}, 8 \mathrm{~d}, 8 \mathrm{f}, 8 \mathrm{~g}, 8 \mathrm{~h})$ and twice in connexion with interaction effects involving age $(8 \mathrm{~b}, 7 \mathrm{~g})$. It is clear from these analyses that individual questions on their own do not differentiate the groups very efficiently so far as smoking habits are concerned. This is not unexpected, because single questions are relatively unreliable; it is only when they are combined in groups that these unreliabilities are reduced owing to the cancelling out of chance errors.

Details of results are given in Table III. Three of the questions relate to extraversion, and in each case non-smokers, as predicted, are more introverted than cigarette smokers. Pipe smokers are relatively introverted, while ex-smokers are similar to medium smokers. On the one question relating to rigidity, non-smokers are the most rigid group, as predicted. On the one question relating to neuroticism, ex-smokers emerge as the most neurotic; smokers as a whole do not appear to be more neurotic than non-smokers.

As regards the two questions concerning which special hypotheses were advanced, the results are as follows. On question $7 \mathrm{~g}$ the hypothesis that non-smokers would use their hands more when talking than would smokers is verified only in the working-class group and not in the middle-class group; the class-group interaction is significant. On question $8 \mathrm{~g}$ the hypothesis that nonsmokers would have more chewing and biting habits than smokers is verified; only ex-smokers excel nonsmokers in this.

\section{Determination of Trait Scores}

The next step in our inquiry was the carrying out of a factor analysis of the correlations between the different personality questions. This was done in order to obtain direct evidence from the data of this inquiry of the feasibility of combining questions into groups. This was a necessary process, since combinations should not be arbitrary, or determined by previous analyses on different groups, and it seemed desirable to check on their applicability to this particular sample.

To ascertain the precise grouping of the inventory items, correlations were calculated between all 31 items, age, class, and smoking. These correlations were then factor-analysed in order to determine the grouping, and four factors were extracted by means of Hotelling's principal components method. This solution was then rotated according to Thurstone's simple structure solution, and revealed three clear-cut factors. The first of these was extraversion, defined by items $5 \mathrm{~d}, 6 \mathrm{f}, 6 \mathrm{~g}, 7 \mathrm{f}$, $8 \mathrm{~b}$, and $8 \mathrm{~h}$. The second factor was one of rigidity, defined by items $6 \mathrm{~b}, 7 \mathrm{a}, 7 \mathrm{~b}, 7 \mathrm{e}, 8 \mathrm{e}, 8 \mathrm{f}$, and $8 \mathrm{i}$. The third factor was one of neuroticism, made up of items 5b, 5c, $5 \mathrm{c}, 5 \mathrm{f}, 6 \mathrm{a}, 6 \mathrm{~d}, 6 \mathrm{e}, 6 \mathrm{~h}, 7 \mathrm{c}, 7 \mathrm{~d}, 8 \mathrm{a}$, and $8 \mathrm{~d}$.

Scores were next calculated for each of these three factors according to the following scheme. For the extraversion and rigidity factors, the answer "Yes" on each of the items defining the factor is scored two points, the answer "No" is scored zero points, and any other answer is scored one point. The same scheme is followed in calculating the neuroticism score, except that for item 6a the answer "No" is scored two points, the answer "Yes" no points, and any other answer one point. These three scores are independent of each other ; when they were correlated over the whole population, neuroticism and extraversion correlated 0.04 , neuroticism and rigidity correlated 0.09 , and extraversion and rigidity correlated 0.07 .

\section{Personality Traits and Smoking Habits}

Mean scores were next calculated on the three factors separately for the various groups of smokers, subdivided by age and sex. Detailed figures may be consulted in the

TABLE III

\begin{tabular}{|c|c|c|c|c|c|c|c|c|c|c|c|c|}
\hline Question & Trait & Non & $\mathrm{L}$ & M & $\mathbf{H}$ & $\mathbf{P}$ & Ex & $\mathrm{F}_{\mathrm{C}}$ & $F_{A}$ & $\mathrm{~F}_{\mathrm{G}}$ & $\mathrm{F}_{\mathrm{AG}}$ & $\mathrm{F}_{\mathrm{CG}}$ \\
\hline $\begin{array}{l}\text { 6f. Would you rate yourself a lively } \\
\text { individual? }\end{array}$ & $\mathbf{E}$ & 529 & 553 & 612 & 624 & 544 & 556 & 0.053 & 0.854 & $4 \cdot 819$ & & \\
\hline $\begin{array}{l}\text { 8d. Are you frequently lost in thought, } \\
\text { even when you're supposed to } \\
\text { be taking part in a conversation } \\
\text { 8f }\end{array}$ & $\mathbf{N}$ & 287 & 308 & 290 & 263 & 301 & 340 & 0.756 & $1 \cdot 323$ & $3 \cdot 100$ & & \\
\hline $\begin{array}{l}\text { 8f. Do you always prefer the familiar, } \\
\text { the safe and sure, to taking } \\
\text { chances, with the new and } \\
\text { untried, } \\
\text { 8g. Do you hre any habits like chsw- }\end{array}$ & $\mathbf{R}$ & 541 & 505 & 492 & 445 & 485 & 455 & $37 \cdot 297$ & $79 \cdot 135$ & 5.652 & & \\
\hline $\begin{array}{l}\text { ing pencils or biting finger-nails } \\
\text { or things like that? }\end{array}$ & S.H. & 116 & 80 & 106 & 76 & 62 & 122 & $0 \cdot 354$ & 19.060 & 3.879 & & \\
\hline $\begin{array}{l}\text { 8h. Are you inclined to be quick and } \\
\text { sure in your actions? }\end{array}$ & $\mathbf{E}$ & 586 & 582 & 588 & 640 & 544 & 588 & $12 \cdot 301$ & $24 \cdot 214$ & $3 \cdot 847$ & & \\
\hline $\begin{array}{l}\text { 8b. Are you happiest in some scheme } \\
\text { that calls for rapid action? }\end{array}$ & $\mathbf{E}$ & 445 & 459 & 488 & 520 & 438 & 478 & $5 \cdot 732$ & $147 \cdot 140$ & $8 \cdot 879$ & $5 \cdot 234$ & \\
\hline $\begin{array}{c}\text { 7g. Do you get so excited that you use } \\
\text { your hands when you talk? }\end{array}$ & S.H. & 196 & 183 & 149 & 197 & 167 & 163 & $2 \cdot 496$ & $137 \cdot 571$ & $5 \cdot 140$ & & $6 \cdot 500$ \\
\hline
\end{tabular}

$E=$ Extraversion. $N=$ Neuroticism. $R=$ Rigidity. S.H. $=$ Special hypothesis. Non $=$ Non-smoker. $L=$ Light smoker. $M=M e d i u m$ smoker. $H=H e a v y$ smoker. $P$ Pipesmoker. Ex $=$ Ex-smoker. $F=F$ ratio. $\mathbf{C}=$ Class. $\mathbf{A}=$ Age. $\mathbf{G}=$ Smoking groups. Figures in italic: significant, $5 \%$. Figures in bold type: significant, $1 \%$. 
original report (Mass Observation, 1959); for reasons of space, only the results of the analysis of variance are here given.

Extraversion.- - The mean scores for non-smokers and light. medium, and heavy smokers are 7.016, 7.133, 7.445, and 7.805, giving a grand mean for these four groups of 7.358. The means increase with an increase in smoking as required by the hypothesis. The mean for the pipe smokers is 6.96, that for ex-smokers 7.27. (No predictions were made for these groups, and consequently they will not be included in the analysis of variance to determine the significance of the relationship between smoking and personality.) An analysis of variance was carried out to determine the significance of the relationship found betwe en extraversion and smoking; the results are given in Table IV. As the

TABLE IV.-Analysis of Variance Table. Extraversion and Smokino

\begin{tabular}{|c|c|c|c|c|}
\hline & $\begin{array}{l}\text { Degrees of } \\
\text { Freedom }\end{array}$ & $\begin{array}{l}\text { Sum of } \\
\text { Squares }\end{array}$ & $\begin{array}{c}\text { Mean } \\
\text { Squares }\end{array}$ & $\begin{array}{c}\text { Variance } \\
\text { Ratio }\end{array}$ \\
\hline $\begin{array}{l}\text { Between groups } \\
\text { Within ", }\end{array}$ & $\begin{array}{r}3 \\
1,556\end{array}$ & $\begin{array}{r}145 \cdot 3082 \\
15,938 \cdot 9510\end{array}$ & $\begin{array}{l}48 \cdot 4361 \\
10 \cdot 2435\end{array}$ & $4 \cdot 728$ \\
\hline Tutal & 1.559 & & & \\
\hline
\end{tabular}

results are significant beyond the $1 \%$ level, the hypothesis may be regarded as established. We can now carry out individual $t$ tests to establish significance of differences between groups. The difference between non-smokers and medium smokers is significant at the $5 \%$ level on a one-tail test; that between non-smokers and heavy smokers is significant at the $5 \%$ level; other differences are not significant.

Rigidity.-The mean scores for non-smokers, light, medium, and heavy smokers are 9.456, 9.285, 9.233, and 8.930 , with a grand mean for these four scores of 9.220 . It will be seen that the scores decline with an increase in smoking as required by the hypothesis. (The mean for pipe smokers is 9.01, that for ex-smokers is 9.23. Again, these groups will be disregarded in calculating the significance of results. as no predictions were made regarding them.) An analysis of variance was carried out, and is reported in Table V. It will be seen that the result is just

Table V.-Analysis of Variance Table. Rigidity and Smoking

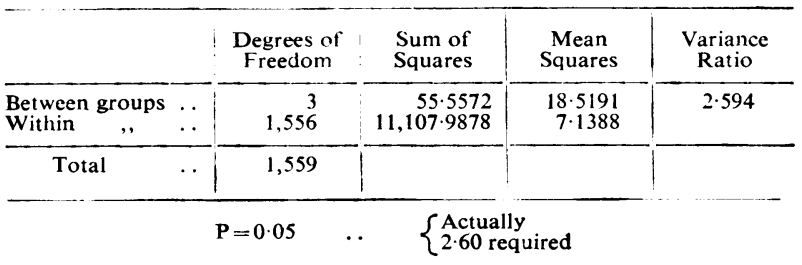

about significant at the $5^{\circ}$ level, and we may therefore calculate $t$ tests to establish significance between sing!e groups. The differences between light and heavy smokers and between non-smokers and heavy smokers are significant at the $5 \%$ and $1 \%$ levels respectively on a one-tail test; none of the other differences are significant. We may consider our hypothesis supported, but not as strongly as in the case of extraversion.

Neuroticism.--The mean scores for non-smokers, light, medium, and heavy smokers are $8.274,8.270,8.445$, and 8.455 , with a grand mean of 8.368. (Pipe smokers have a mean of 8.22 and ex-smokers one of 8.43 . These two scores not having been predicted are not included in the analysis of variance.) An analysis of variance was performed, and is given in Table VI. It will be seen that while the score on neuroticism increased as predicted with the amount of smoking, the results are not significant. It is possible that
TABLE VI-Analysis of Variance Table. Neuroticism anid Smoking

\begin{tabular}{|c|c|c|c|c|}
\hline & $\begin{array}{l}\text { Degrees of } \\
\text { Freedom }\end{array}$ & $\begin{array}{l}\text { Sum of } \\
\text { Squares }\end{array}$ & $\begin{array}{c}\text { Mean } \\
\text { Squares }\end{array}$ & $\begin{array}{c}\text { Variance } \\
\text { Ratio }\end{array}$ \\
\hline $\begin{array}{l}\text { Between groups } \\
\text { Within }, "\end{array}$ & $\begin{array}{r}3 \\
1,556\end{array}$ & $\begin{array}{r}12.4218 \\
46,998.7323\end{array}$ & $\begin{array}{r}4 \cdot 1406 \\
30 \cdot 2048\end{array}$ & 0.137 \\
\hline Total & 1,559 & & & \\
\hline
\end{tabular}

with an increase in numbers significance might be attained, but this is by no means certain.

The results strongly support our hypothesis regarding extraverts being heavier smokers than introverts; they weakly support our hypothesis about non-smokers being more rigid than smokers; they fail to support at an adequate level of significance the hypothesis that smokers

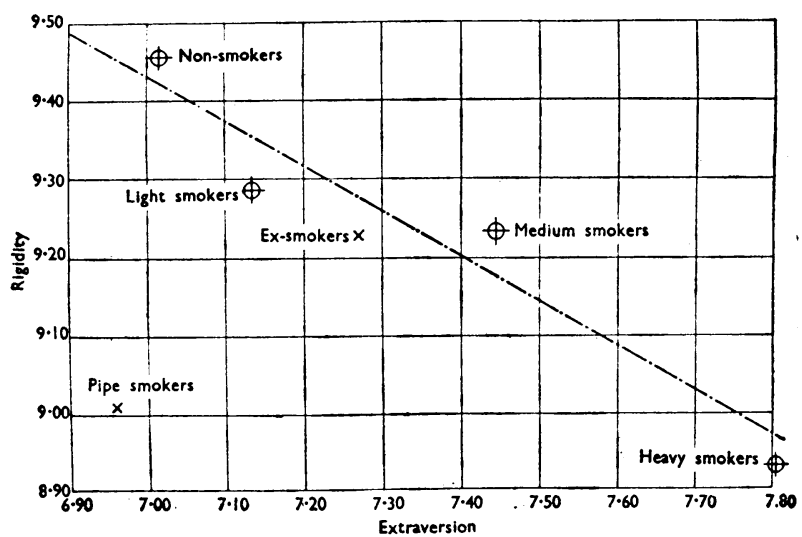

Graph showing relation between smoking habits and rigidity and extraversion.

are more neurotic than non-smokers. A Graph has been prepared to show the relationship between smoking habits on the one hand and rigidity and extraversion on the other. The linearity of regression would seem to lend support to our interpretation of the results.

\section{Discussion}

The results of this study support the hypothesis that genotypic personality differences are related to smoking habits. (It should be borne in mind that there is some evidence from twin studies that extraversion is in part determined by genetic factors (Eysenck, 1957), and this personality trait has also been related to certain biological properties of the cortex (Eysenck, 1957.) They do not prove that smoking does not cause lung cancer, but they make more reasonable than had previously been the case the proposition that both smoking and cancer may be causally related to certain underlying genotypic factors. Perhaps the most appealing hypothesis is that extraverts live at an accelerated rate, drinking harder, smoking harder, living more irregular lives, staying up longer, and generally "living it up" more. They may thus (a) lower their resistance to disease, and (b) expose themselves more to conditions which may directly cause the disease in question. This would agreo well with the findings of Pearl (1928) and of Hammond and Horn (1954) that the general death rate among smokers is similar to that of non-smokers of a more advanced age. (It may be that, in addition, non-smokers are biologically self-protective, as Berkson (1958) hypothesizes, and that biologically this is correlated with robustness in meeting mortal stress from disease 
generally. Such an additional hypothesis is not strictly necessary, and does not appear very likely to us.)

Some such theory as that outlined above would account for all those features of the evidence which had caused difficulties to the proponents of the "direct action" hypothesis. Inhaling now becomes irrelevant, unless it can be shown to correlate with introversion; it does not provide any obstacle to our theory. The relationship between smoking and non-cancerous diseases also finds an explanation. Lastly, the fact that pipe smokers are not particularly prone to lung cancer finds an easy explanation in terms of their quite different personality structure; a glance at the graph will show that they are even more introverted than are nonsmokers. Their failure to develop lung cancer thus finds a much more obvious explanation than the purely ad hoc hypotheses to which Fisher (1959) has drawn attention rather scathingly.

Certain consequences follow from these findings with respect to future research. In the first place, the study should be repeated with more extensive questionaries, constructed specifically to follow up the suggestive findings reported above. In the second place, questions ought to be asked about inhaling, in order to test the possibility that introverts inhale more than extraverts who smoke the same amount. Last, and much the most important, a direct study should be undertaken of the personality of victims of lung cancer; if they could be shown to be more extraverted than a carefully chosen control group the theory here advanced would receive considerable support.

\section{Summary}

The theory that smoking causes lung cancer has received much support. The investigation here reported was carried out to test certain deductions from an alternative theory, according to which both smoking and lung cancer are symptoms of one and the same underlying factor, related to genotypic differences between smokers and non-smokers. It was hypothesized that cigarette smokers would be (1) more extraverted, (2) less rigid, and (3) more neurotic than non-smokers. An empirical investigation of 2,360 male subjects, selected so as to conform to a predetermined sampling scheme, and stratified according to age, social class, and smoking habits, strongly confirmed the first of these hypotheses, weakly confirmed the second, and failed to confirm the third. Pipe smokers were found to be quite different from cigarette smokers from the point of view of personality, being the most introverted group studied. On the whole the data confirm the view that genotypic differences exist between smokers and non-smokers, and between cigarette smokers and pipe smokers.

\section{APPENDIX}

5a. Do you find you have to take care of your health or have you a naturally strong constitution? Just say "yes" or " no" to all these points, whichever you think correct. You need not think deeply about it.

b. Do you use up more energy than the average person in getting things done?

c. Do you sometimes feel happy, sometimes depressed, without any apparent reason?

d. Do you prefer action to planning for action?

e. Do you get excited when you argue ?

f. Are you inclined to be moody ?

6a. Now, can you relax easily when sitting or lying down? b. Do you become so much absorbed in whatever you're doing that you don't like to be interrupted and have to change to something new ?

c. Do you easily show it when you feel sympathy, delight, sorrow, or anger?

d. And if you are feeling angry, sympathetic, or jealous, do you sometimes do things that you regret later on ?

e. Are you easily startled when things happen unexpectedly ?

f. Would you rate yourself a lively individual ?

g. Do you usually take the initiative in making new friends ?

h. Are you sometimes bubbling over with energy and sometimes very sluggish ?

7a. Do you feel you can really enjoy yourself with friends when you've done your duty and finished whatever task you have in mind?

b. Do you remain outwardly calm when people around you are moved and excited ?

c. Does your mind often wander when you are trying to concentrate?

d. Do you have frequent ups and downs in mood either with or without apparent cause ?

e. Before going on a trip do you always plan well in advance, decide how you're going and what you're going to do and then feel reluctant to make changes?

f. Would you be very unhappy if you were prevented from making numerous social contacts ?

g. Do you get so excited that you use your hands when you talk ?

h. Do you suffer from frequent sleeplessness ?

8a. Are you easily upset when you're disturbed in something you're concentrating on ?

b. Are you happiest in some scheme that calls for rapid action?

c. Do you ever get a lump in your throat when you read a sad story or see an emotional film ?

d. Are you frequently lost in thought, even when you're supposed to be taking part in a conversation?

e. Do you think a person who does not live up to the standards he sets himself does not deserve sympathy?

f. Do you always prefer the familiar, the safe and sure, to taking chances with the new and untried ?

g. And do you have any habits like chewing pencils or biting finger-nails or things like that ?

h. Are you inclined to be quick and sure in your actions ?

i. Finally, do you feel one should never take the second-best and only buy the most solid or lasting things are else go without?

\section{REFERENCES}

Berkson, J. (1958). J. Amer. statist. Ass., 53, 28.

Doll, R., and Hill, A. B. (1952). Brit. med. J., 2, 1271

- (1954). Ibid., 1, 1451

(1956). Ibid., 2, 1071

Eysenck, H. J. (1947). Dimensions of Personality. Kegan Paul, Trench, Trubner, London.

(1952). The Scientific Study of Personality. Routledge and Kegan Paul, London.

(1957). The Dynamics of Anxiety and Hysteria. Routledge and Kegan Paul, London.

(1959). Manual of the Maudsley Personality Inventory. Univ. of London Press.

(1960a). The Structure of Human Personality, 2nd ed. Methuen, London.

(Editor) (1960b). Experiments in Personality, 2 vols. Routledge and Kegan Paul, London.

Fisher, R. A. (1959). Smoking-the Cancer Controversy. Oliver and Boyd, Edinburgh.

Hammond, E. G., and Horn, D. (1954). J. Amer. med. Ass., 155, 1316.

McArthur, C., Waldron, E., and Dickinson, J. (1958). J. abnorm. soc. Psychol., 56, 267.

Mass-Observation (1959). Personality Factors and Smoking. Mass-Observation, London.

Nigniewitzky, R. D. (1955). A Statistical Study of Rigidity as a Personality Variable. Univ. of London: Unpublished thesis.

Pearl, R. (1928). The Rate of Living. Knopf, New York.

Simpson, W. J. (1957). Amer. J. Obstet. Gynec., 73, 808.

Stocks, P., and Campbell, J. M. (1955). Brit. med. J., 2, 923.

Todd, G. F., and Laws, J. T. (1958). Tobacco Manufacturers Standing Committee, Research Papers, No. 2. 